\title{
Cytotoxic T lymphocytes: the future of cancer stem cell eradication?
}

\section{"Cancer stem-like cells/cancer-initiating cells are immunogenic to cytotoxic $T$ lymphocytes and express several tumor-associated antigens that can be recognized by cytotoxic T lymphocytes."}

\section{KEYWORDS: cancer stem cell $₫$ cytotoxic T lymphocyte $\approx$ immunotherapy $\approx$ tumor antigen}

Cancer stem-like cells (CSCs)/cancer-initiating cells (CICs) are a hot topic in cancer research since they are highly tumorigenic and are resistant to standard cancer therapies. Recent studies have revealed that cancer immunotherapy is a possible and promising candidate to target CSCs/CICs. Among the various immunological effector cells, cytotoxic T lymphocytes (CTLs) are a good candidate for CSC/CIC-targeted immunotherapy as CTLs are antigen-specific effector cells. In this article, we summarize advances in studies on CTLs and discuss the future of CSC/CIC-targeted therapy.

Since the first identification of leukemia stem cells from an acute myeloid leukemia sample, studies of CSCs/CICs have made great advances [1]. CSCs/CICs are a small population of cancer cells that have tumor-initiating, self-renewal and differentiation abilities. Recent studies have revealed that CSCs/CICs are resistant to cancer therapies because they are in a quiescent cellcycle state, they express high levels of transporters and apoptosis inhibitors, and they express low levels of reactive oxygen species. CSCs are therefore regarded as major causes of cancer recurrence, distant metastasis and treatment resistance; studies of CSCs/CICs have been focusing on how CSCs/CICs can be targeted efficiently.

\section{Cancer immunotherapy: a possible option for CSC/CIC-targeted therapy}

Cancer immunotherapy is expected to become the fourth main cancer therapy following surgery, chemotherapy and radiotherapy. Several cancer immunotherapy protocols have been tested in clinical trials, and the first cancer immunotherapy drug Provenge ${ }^{\circledR}$ (Dendreon, WA, USA) was approved by the US FDA in 2010 for treatment of advanced prostate cancer. It had not been clear whether the immune system can recognize therapy-resistant CSCs/CICs, but recent studies have revealed that both the innate and acquired immune system can recognize CSCs/CICs [2]. CTLs, NK cells, $\gamma \delta \mathrm{T}$ cells and antibodies have been shown to be able to target CSCs/CICs. CTLs, NK cells and $\gamma \delta$ T cells kill target cells by cytotoxic granules including perforin and granzymes, therefore treatment-resistant CSCs/CICs would be susceptible to death. CTLs are a key player in the aquired immune system and recognize target cells in an antigen-specific manner. On the other hand, NK cells and $\gamma \delta \mathrm{T}$ cells are players in the innate immune system and are not specific for antigens. Therefore, CTLs might be useful for CSC/CIC-targeted immune therapy.

\section{CTLs: key player for eradicating CSCs/CICs}

Since CTLs recognize antigenic peptides presented by MHCs, it is essential that CSCs/CICs express both MHCs and tumor-associated antigens (TAAs). A previous study revealed that colon cancer stem cells isolated as side population cells express MHC molecules at levels similar to those in non-CSCs/CICs [3]. In a recent study, Carbone's group found that colon CSCs/CICs derived as spheroid cultures express lower levels of MHC molecules than parental colon tumor cells, which enables NK cells to recognize CSCs/CICs [4]. They reported that NK cells could recognize colon CSCs/CICs because they express higher levels of ligands of NK cell-activating receptors, including NKp30 and NKp44. However, colon CSCs/CICs used in the study expressed MHC molecules to some extent, which may be sufficient to be recognized by CTLs. In fact, we and other groups have reported that CSCs/CICs can be recognized by CTLs $[3,5,6]$ and CSCs/CICs might

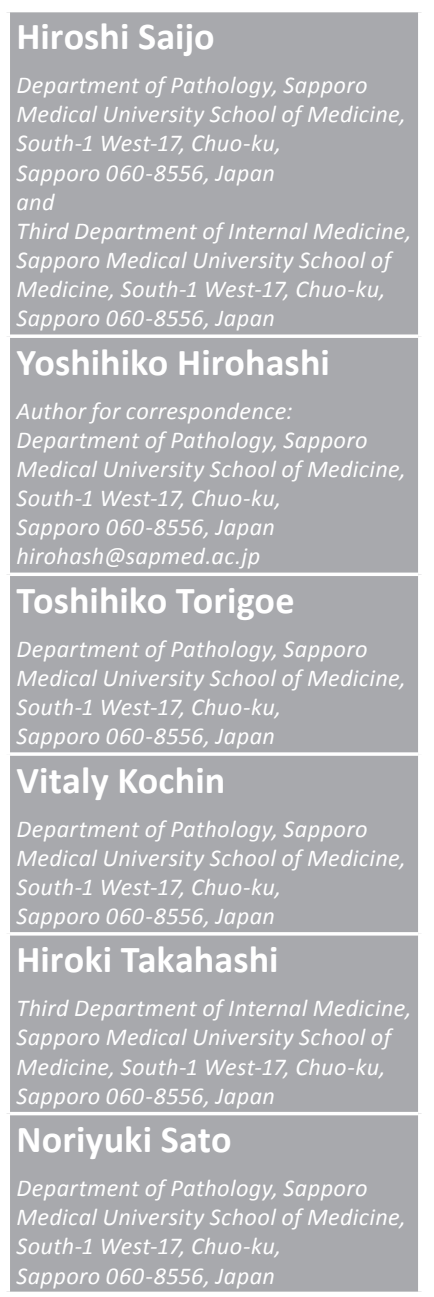

Future $\mathrm{fSS}$ 
therefore express sufficient levels of MHCs to be recognized by CTLs.

CSCs/CICs have been shown to express several TAAs. We previously classified TAAs into three groups according to expression profiles in CSCs/CICs and non-CSCs/CICs [7]. These three groups are: CSC/CIC antigens, which are expressed preferentially in CSCs/CICs; shared antigens, which are expressed in both CSCs/CICs and non-CSCs/CICs; and non-CSC/CIC antigens, which are expressed preferentially in nonCSCs/CICs. Thus, CSC/CIC antigens and shared antigens are expressed in CSCs/CICs. The CSC/CIC antigens so far reported include MAGEA3, MAGEA4, DNAJB8, SOX2, OCT3/4, BMI1 and ALDH1A1 [6,8-11]. SOX2, OCT3/4 and ALDH1A1 are also expressed in the normal stem cell fraction, whereas MAGEA3, MAGEA4 and DNAJB8 are cancer testis (CT) antigens that are expressed in CSCs/CICs and normal testis cells, but not in normal stem cells. SOX2, OCT3/4 and ALDH1A1 can be candidates for CSC/CIC-targeted immunotherapy; however, there is a risk of also targeting normal stem cells, which may make the patient's condition severe. Therefore, CT antigens may be good candidates for CSC/CIC-targeted immunotherapy. Interestingly, large numbers of CT antigens are preferentially expressed in CSCs/CICs [12]. The testis is an 'immunologically privileged organ', and CT antigens are regarded as immunogenic TAAs [13]. The biological significance of testis gene products in CSCs/CICs is still elusive; however, they should be better candidates for CSC/CIC-targeted immunotherapy as they are not expressed in normal stem cells.

\section{"...both the innate and acquired immune system can recognize cancer stem-like cells/cancer-initiating cells."}

Since both CSC/CIC antigens and shared antigens are expressed in CSCs/CICs, it raises the question, which one is better for targeting CSCs/CICs? We have identified a novel CSC/CIC antigen, DNAJB8, which is expressed in kidney CSCs/CICs [11]. To answer the above question, we compared the potency of DNAJB8 with that of survivin, which is a well-established TAA and is a shared antigen [14]. Interestingly, DNAJB8 was more potent than survivin in a tumor prophylactic DNA vaccination model. However, it may be too early to reach a conclusion; these results suggest that CSC/CIC antigens are better at targeting cancers than shared antigens. Several other studies have demonstrated the potency of CSC/CIC-targeted immunotherapy by in vivo animal models $[5,6,15]$. Therefore, CSC/CIC-targeted immunotherapy using CSC/CIC antigens is a feasible and promising approach.

\section{"...cancer stem-like cell/cancer-initiating cell antigens are better at targeting cancers than shared antigens."}

There are several molecular mechanisms by which CSCs/CICs may escape from CTLs. Heinberger's group reported that glioma stem cells express high levels of immunosuppressive molecules, including B7-H1 and soluble galectin-3 [16]. Kim's group reported that CSCs/CICs express high levels of NANOG, which induces CTL resistance by activation of Nanog/Tcl1a/ Akt signaling [17]. The results of these two studies demonstrate the molecular mechanisms of suppression of CTL induction phase and suppression of CTL effector phase, respectively. Since CSCs/CICs express high levels of CT antigens, which are regarded as highly immunogenic TAAs, CSCs/CICs must be a relatively immunogenic cancer cell population. However, CSCs/CICs in clinical cancer specimens survive immune pressure, indicating that there are mechanisms for CSCs/CICs to escape the immune system. These two mechanisms may be aspects of CSC/CIC immune escape. Therefore, to overcome the problem of immune escape of CSCs/CICs and target CSCs/CICs in cancer immunotherapy, an immune potentiator (adjuvant) may be necessary to achieve significant antitumor effects.

\section{Conclusion}

CSCs/CICs are immunogenic to CTLs and express several TAAs that can be recognized by CTLs. CSC/CIC-targeted immunotherapy using these TAAs might be feasible. Antigenic peptide vaccination and CTL adoptive transfer are possible approaches to target CSCs/CICs. In the near future, chemotherapy- and radiotherapyresistant CSCs/CICs may be able to be targeted by CTLs in clinical settings.

\section{Financial \& competing interests disclosure}

This work was supported by Grants-in-Aid for Scientific Research from the Ministry of Education, Culture, Sports, Science and Technology of Japan (grant numbers 16209013, 17016061 and 15659097), for Practical Application Research from the Japan Science and Technology Agency, and for Cancer Research (15-17 and 19-14) from the Ministry of Health, Labor and Welfare of Japan, Ono 
Cancer Research Fund (to N Sato) and Takeda Science Foundation (to Y Hirohashi). This work was supported in part by the National Cancer Center Research and Development Fund (23-A-44). The authors have no other relevant affiliations or financial involvement with any

\section{References}

1 Clevers $\mathrm{H}$. The cancer stem cell: premises, promises and challenges. Nat. Med. 17 , 313-319 (2011).

2 Hirohashi $Y$, Torigoe $T$, Inoda $S$ et al. Immune response against tumor antigens expressed on human cancer stem-like cells/tumor-initiating cells. Immunotherapy 2, 201-211 (2010).

3 Inoda S, Hirohashi Y, Torigoe T et al. Cytotoxic T lymphocytes efficiently recognize human colon cancer stem-like cells. $A m$. J. Pathol. 178, 1805-1813 (2011).

4 Tallerico R, Todaro M, Di Franco S et al. Human NK cells selective targeting of colon cancer-initiating cells: a role for natural cytotoxicity receptors and MHC class I molecules. J. Immunol. 190, 2381-2390 (2013).

5 Xu Q, Liu G, Yuan X et al. Antigen-specific T-cell response from dendritic cell vaccination using cancer stem-like cell-associated antigens. Stem Cells 27, 1734-1740 (2009).

6 Visus C, Wang Y, Lozano-Leon A et al. Targeting ALDH (bright) human carcinoma- organization or entity with a financial interest in or financial conflict with the subject matter or materials discussed in the manuscript apart from those disclosed.

No writing assistance was utilized in the production of this manuscript. initiating cells with ALDH1A1-specific CD8 ${ }^{+}$ T cells. Clin. Cancer Res. 17, 6174-6184 (2011).

7 Hirohashi $Y$, Torigoe T, Inoda S, Morita R, Kochin V, Sato N. Cytotoxic T lymphocytes: sniping cancer stem cells. Oncoimmunology 1, 123-125 (2012).

8 Schmitz M, Temme A, Senner V et al. Identification of SOX2 as a novel gliomaassociated antigen and potential target for T cell-based immunotherapy. Br. J. Cancer 96, 1293-1301 (2007).

9 Steele JC, Torr EE, Noakes KL et al. The polycomb group proteins, BMI-1 and EZH2, are tumour-associated antigens. Br. J. Cancer 95, 1202-1211 (2006).

10 Dhodapkar KM, Feldman D, Matthews P et al. Natural immunity to pluripotency antigen OCT4 in humans. Proc. Natl Acad. Sci. USA 107, 8718-8723 (2010).

11 Nishizawa S, Hirohashi Y, Torigoe T et al. HSP DNAJB8 controls tumor-initiating ability in renal cancer stem-like cells. Cancer Res. 72, 2844-2854 (2012).
12 Yamada R, Takahashi A, Torigoe T et al. Preferential expression of cancer/testis (CT) genes in cancer stem-like cells: proposal of a novel sub-category, cancer/testis/stem (CTS) gene. Tissue Antigens doi:10.1111/tan.12113. (2013) (Epub ahead of print).

13 Scanlan MJ, Simpson AJ, Old LJ. The cancer/testis genes: review, standardization, and commentary. Cancer Immun. 4, 1 (2004).

14 Andersen MH, Svane IM, Becker JC, Straten PT. The universal character of the tumor-associated antigen survivin. Clin. Cancer Res. 13, 5991-5994 (2007).

15 Ning N, Pan Q, Zheng F et al. Cancer stem cell vaccination confers significant antitumor immunity. Cancer Res. 72, 1853-1864 (2012).

16 Wei J, Barr J, Kong LY et al. Gliomaassociated cancer-initiating cells induce immunosuppression. Clin. Cancer Res. 16, 461-473 (2010).

17 Noh KH, Kim BW, Song KH et al. Nanog signaling in cancer promotes stem-like phenotype and immune evasion. J. Clin. Invest. 22(11), 4077-4093 (2012). 